\title{
A Continuous $d$-Step Conjecture for Polytopes
}

\author{
Antoine Deza · Tamás Terlaky • Yuriy Zinchenko
}

Received: 11 September 2007 / Revised: 25 May 2008 / Accepted: 30 May 2008 /

Published online: 1 July 2008

(C) Springer Science+Business Media, LLC 2008

\begin{abstract}
The curvature of a polytope, defined as the largest possible total curvature of the associated central path, can be regarded as a continuous analogue of its diameter. We prove an analogue of the result of Klee and Walkup. Namely, we show that if the order of the curvature is less than the dimension $d$ for all polytopes defined by $2 d$ inequalities and for all $d$, then the order of the curvature is less that the number of inequalities for all polytopes.
\end{abstract}

Keywords Polytopes $\cdot$ Diameter $\cdot$ Hirsch conjecture $\cdot d$-Step conjecture $\cdot$ Central path $\cdot$ Curvature

\section{Continuous Analogue of the Conjecture of Hirsch}

Let $P$ be a full-dimensional convex polyhedron defined by $n$ inequalities in dimension $d$. Given a pair of vertices $v=\left\{v_{1}, v_{2}\right\}$ of $P$, let $\delta^{v}(P)$ denote the smallest number such that $v_{1}$ and $v_{2}$ can be connected by a path with at most $\delta^{v}(P)$ edges. Considering the maximum of $\delta^{v}(P)$ over all possible pairs of vertices, we obtain the diameter $\delta(P)$, that is, the smallest number such that any two vertices of the polyhedron $P$ can be connected by a path with at most $\delta(P)$ edges. The conjecture of Hirsch, formulated in 1957 and reported in [1], states that the diameter of a polyhedron defined by $n$ inequalities in dimension $d$ is not greater than $n-d$. The conjecture does

\footnotetext{
A. Deza · T. Terlaky · Y. Zinchenko $(\bowtie)$

Advanced Optimization Laboratory, Department of Computing and Software, School of Computational Engineering and Science, McMaster University, 1280 Main Street West, Hamilton, Ontario L8S 4K1, Canada e-mail: zinchen@mcmaster.ca

A. Deza

e-mail: deza@mcmaster.ca

T. Terlaky

e-mail: terlaky@mcmaster.ca
} 
not hold for unbounded polyhedra. A polytope is a bounded polyhedron. No polynomial bound is known for the diameter of a polytope; the best one is $2 n^{\log (d)+1}$, see Kalai and Kleitman [8].

Conjecture 1.1 (Conjecture of Hirsch for polytopes) The diameter of a polytope defined by $n$ inequalities in dimension $d$ is not greater than $n-d$.

We consider the following continuous analogue of the diameter introduced in [4]: the largest possible total curvature of the associated central path. We first recall the definitions of the central path and total curvature. For a polytope $P=\{x: A x \geq$ $b$ w with $A \in \mathbb{R}^{n \times d}$, the central path corresponding to $\min \left\{c^{T} x: x \in P\right\}$ is a set of minimizers of $\min \left\{c^{T} x+\mu f(x): x \in P\right\}$ for $\mu \in(0, \infty)$, where $f(x)$ is the so-called strongly non-degenerate self-concordant barrier function [10] for $P$, and throughout this paper, $f(x)=-\sum_{i=1}^{n} \ln \left(A_{i} x-b_{i}\right)$ is the standard logarithmic barrier function. As a consequence of strict convexity of the logarithmic barrier function, the central path is unique. It is known that the central path is an analytic curve [12] and thus, in particular, is fully determined by any of its arbitrary small continuous segment or all of its derivatives at any a single point. Conversely, the analyticity of the central path should allow us to reconstruct the full description of a polytope $P$ up to its orientation given by the objective vector $c$ and possibly some equivalence relationship between the polytopes based on the central path.

Intuitively, the total curvature [13] is a measure of how far off a certain curve is from being a straight line. Let $\psi:[\alpha, \beta] \rightarrow \mathbb{R}^{d}$ be a $C^{2}((\alpha-\varepsilon, \beta+\varepsilon))$ map for some $\varepsilon>0$ with a nonzero derivative in $[\alpha, \beta]$. Denote its arc length by $l(t)=$ $\int_{\alpha}^{t}\|\dot{\psi}(\tau)\| d \tau$, its parametrization by the arc length by $\psi_{\text {arc }}=\psi \circ l^{-1}:[0, l(\beta)] \rightarrow$ $\mathbb{R}^{d}$, and its curvature at the point $t$ by $\kappa(t)=\ddot{\psi}_{\text {arc }}(t)$. The total curvature is defined as $\int_{0}^{l(\beta)}\|\kappa(t)\| d t$. The requirement $\dot{\psi} \neq 0$ insures that any given segment of the curve is traversed only once and allows one to define a curvature at any point on the curve.

Let $\lambda^{c}(P)$ denote the total curvature of the central path corresponding to the linear optimization problem $\min \left\{c^{T} x: x \in P\right\}$. The quantity $\lambda^{c}(P)$ can be regarded as a continuous analogue of $\delta^{v}(P)$, the edge-length of the shortest path between a pair of vertices. Considering the largest $\lambda^{c}(P)$ over all possible $c$, we obtain the quantity $\lambda(P)$ referred to as the curvature of a polytope. The following analogue of the conjecture of Hirsch was proposed in [4].

Conjecture 1.2 (Continuous analogue of the conjecture of Hirsch) The order of the curvature of a polytope defined by $n$ inequalities in dimension $d$ is $n$.

Holt and Klee [6] showed that, for $n>d \geq 13$, the conjecture of Hirsch is tight. Fritzsche and Holt [5] extended the result to $n>d \geq 8$. The continuous analogue would be that Conjecture 1.2 is the best possible. In [3] the authors showed that a redundant Klee-Minty $d$-cube $\mathcal{C}$ satisfies $\lambda(\mathcal{C}) \geq\left(\frac{3}{2}\right)^{d}$, and in [4] provided a family of $d$-dimensional polytopes $\mathcal{P}$ defined by $n>2 d$ non-redundant inequalities satisfying $\liminf _{n \rightarrow \infty} \lambda(\mathcal{P}) / n \geq \pi$ for a fixed $d$. In other words, the continuous analogue of the result of Holt and Klee holds. 
Proposition 1.1 (Continuous analogue of the result of Holt and Klee) The conjectured order $n$ of the curvature is attained.

An exponential upper bound on the curvature of a polytope is provided by the result of Dedieu, Malajovich, and Shub [2]. Consider a simple arrangement formed by $n$ hyperplanes in dimension $d$; we recall that an arrangement is called simple if $n \geq d+1$ and if any $d$ hyperplanes intersect at a unique distinct point. The number $I$ of bounded cells $P_{i}, i=1, \ldots, I$, of the arrangement, i.e., closures of the bounded connected components of the complement to the hyperplanes, satisfies $I=\left(\begin{array}{c}n-1 \\ d\end{array}\right)$. Dedieu, Malajovich, and Shub [2] demonstrated that $\sum_{i=1}^{i=I} \lambda^{c}\left(P_{i}\right) / I \leq 2 \pi d$ for any fixed $c$. It immediately implies that $\lambda^{c}(P) \leq 2 \pi d\left(\begin{array}{c}n-1 \\ d\end{array}\right)<2 \pi d n^{d-1}<2 \pi n^{d}$ for any $c$, i.e., $\lambda(P) \leq 2 \pi n^{d}$.

Proposition 1.2 (Exponential upper bound on the curvature) The curvature of a polytope defined by $n$ inequalities in dimension $d$ is not greater than $2 \pi n^{d}$.

The special case of $n=2 d$ of the conjecture of Hirsch is known as the $d$-step conjecture, and its continuous analogue is:

Conjecture 1.3 (Continuous analogue of the $d$-step conjecture) The order of the curvature of a polytope defined by $2 d$ inequalities in dimension $d$ is $d$.

It has been shown by Klee and Walkup [9] that the special case $n=2 d$ for all $d$ is equivalent to the conjecture of Hirsch. We prove a continuous analogue of the result of Klee and Walkup, namely, we show in Sect. 2 that Conjectures 1.2 and 1.3 are equivalent.

To motivate the study of the diameter and the total curvature, consider solving a linear optimization problem as above. Roughly speaking, the diameter may be thought of as a lower bound on the number of pivots for a simplex method for the worst possible objective vector $c$, while the total curvature of the central path gives an insight into the performance of a path-following interior-point method (straight line is easy to follow). For polytopes and arrangements, respectively central path and linear optimization, we refer to the books of Grünbaum [7] and Ziegler [14], respectively Renegar [10] and Roos et al. [11].

\section{A Continuous Analogue of the Result of Klee and Walkup}

Following the analogy with the diameter, let $\Lambda(d, n)$ be the largest total curvature $\lambda(P)$ of the central path over all polytopes $P$ defined by $n$ inequalities in dimension $d$. Klee and Walkup [9] showed that for the Hirsch conjecture, the special case where the number of inequalities is twice the dimension is equivalent to the general case. We prove that the same holds for the continuous Hirsch conjecture.

Theorem 2.1 (Continuous analogue of the result of Klee and Walkup) The continuous Hirsch conjecture is equivalent to the continuous $d$-step conjecture; that is, if $\Lambda(d, 2 d)=\mathcal{O}(d)$ for all $d$, then $\Lambda(d, n)=\mathcal{O}(n)$. 
Remark 2.1 In Theorem 2.1, the constant 2 may be replaced by any integer $k>1$, i.e, if $\Lambda(d, k d)=\mathcal{O}(d)$ for all $d$, then $\Lambda(d, n)=\mathcal{O}(n)$.

The rest of Sect. 2 is devoted to the proof of Theorem 2.1. Suppose that $\Lambda(d, 2 d) \leq$ $2 K d, d \geq 2$. Consider $\min \left\{c^{T} x: x \in P\right\}$, where $P=\{x: A x \geq b\}$ with $A \in \mathbb{R}^{n \times d}$ and $\mathcal{P}$ and $\chi$ respectively denote the associated central path and analytic center, i.e., the minimizer of the standard logarithmic barrier $f(x)$ over $P$. The two cases $d<n<2 d$ and $n>2 d$ are considered separately. Denote by $\mathbf{0}$ and $\mathbf{1}$ the vectors of all zeros and of all ones, and by int $P$ the interior of $P$. We may assume that $P$ is full-dimensional, for if not, we may reduce the problem dimension to satisfy the assumption. Note that $A$ is full-rank since $P$ is bounded.

Case $d<n<2 d$ : without loss of generality assume that $c=(1,0, \ldots, 0) \in \mathbb{R}^{d}$ and denote by $x_{1}^{*}$ the optimal value of $\min \left\{c^{T} x: x \in P\right\}$. Consider $\min \left\{c^{T} x: x \in \widetilde{P}\right\}$, where $\widetilde{P}=\{x: \widetilde{A} x \geq \widetilde{b}\}$ with $\widetilde{A} \in \mathbb{R}^{2 d, d}$ and $\widetilde{b} \in \mathbb{R}^{2 d}$ are given by:

$$
\begin{gathered}
\widetilde{A}_{i, j}= \begin{cases}A_{i, j} & \text { for } i=1, \ldots, n \text { and } j=1, \ldots, d, \\
1 & \text { for } i=n+1, \ldots, 2 d \text { and } j=1, \\
0 & \text { for } i=n+1, \ldots, 2 d \text { and } j=2, \ldots, d,\end{cases} \\
\widetilde{b}_{i}= \begin{cases}b_{i} & \text { for } i=1, \ldots, n, \\
x_{1}^{*}-1 & \text { for } i=n+1, \ldots, 2 d,\end{cases}
\end{gathered}
$$

and $\widetilde{\mathcal{P}}$ denotes the associated central path, see Fig. 1(a). Recall that the central path may be parameterized as the collection of the analytic centers of the level sets between the optimal solution and $\chi$; that is, $\mathcal{P}$ is the set of minimizers of $f(x)$ over $P \cap\left\{x: c^{T} x=\omega\right\}$, where $\omega \in\left(x_{1}^{*}, \chi_{1}\right)$. Therefore, we have $\mathcal{P} \subseteq \widetilde{\mathcal{P}}$ and consequently $\lambda^{c}(P) \leq \lambda^{c}(\widetilde{P})$. As $\widetilde{P}$ is defined by $2 d$ inequalities in dimension $d$, we have $\lambda^{c}(\widetilde{P}) \leq 2 K d$, and thus, for $d<n<2 d, \lambda^{c}(P) \leq 2 K d$, that is, $\Lambda(d, n)=\mathcal{O}(n)$.

Case $n>2 d$ : without loss of generality assume that $\chi=\mathbf{0}$. Consider $\min \left\{(c,-\theta)^{T}\right.$ $(x, y):(x, y) \in \widetilde{P}\}$, where $\widetilde{P}=\left\{(x, y) \in \mathbb{R}^{d} \times \mathbb{R}: A x-b y \geq 0, y \leq 1\right\}$ with the associated central path $\widetilde{\mathcal{P}}$. In particular, if the definition of $P$ is non-redundant, $\widetilde{P}$ is the $(d+1)$-dimensional flipped pyramid with base $P \times\{1\}$ and apex $(\chi, 0)=\mathbf{0}$. We show that, for large enough value of $\theta \gg\|c\|$, the central path $\mathcal{P}$ of the original optimization problem may be well approximated by a segment of the central path $\widetilde{\mathcal{P}}$ so that the total curvature of $\mathcal{P}$ is bounded from above by the total curvature of $\widetilde{\mathcal{P}}$. Intuitively, by choosing $\theta$ large enough we should be able to force $\widetilde{\mathcal{P}}$ to first follow almost a straight line from the analytic center of $\widetilde{P}$ to the face containing $P \times\{1\}$, and once $\widetilde{\mathcal{P}}$ is forced almost onto this face, the path should look just like the central path $\mathcal{P}$ for $\min \left\{c^{T} x: x \in P\right\}$ in one-less dimension, see Fig. 1(b). Consequently, we argue that the total curvature of $\mathcal{P}$ may not be less than that of $\widetilde{\mathcal{P}}$.

Denote $s=A x-b y$, and let $S$ be the diagonal matrix with $S_{i i}=s_{i}, i=$ $1, \ldots, d$. Recall that the barrier functions $\widetilde{f}(x, y):$ int $\widetilde{P} \rightarrow \mathbb{R}$ and $f(x):$ int $P \rightarrow \mathbb{R}$ giving rise to the central paths $\widetilde{\mathcal{P}}=\left\{(\tilde{x}, \tilde{y})(v)=\arg \min _{(x, y)} v(c,-\theta)^{T}(x, y)+\right.$ $\widetilde{f}(x, y), v \in[0, \infty)\}$ and $\mathcal{P}=\left\{x(v)=\arg \min _{x} v c^{T} x+f(x), v \in[0, \infty)\right\}$ are 
Fig. 1 An illustration for the proof of Theorem 2.1 for $d=2$ : (a) $2 d>n=3$, (b) $2 d<n=5$

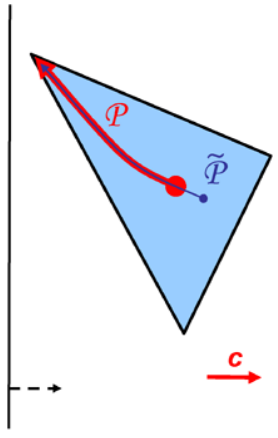

(a)

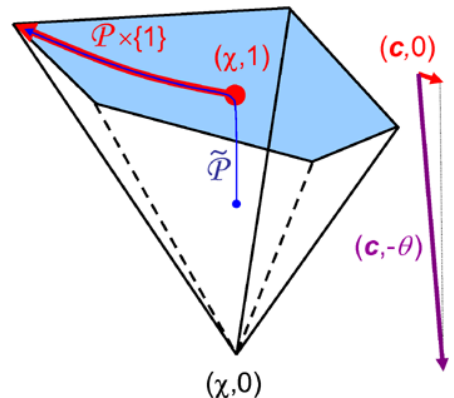

(b)

$\tilde{f}(x, y)=-\sum_{i=1}^{n} \ln s_{i}-\ln (1-y)$ and $f(x)=-\left.\sum_{i=1}^{n} \ln s_{i}\right|_{y=1}$. The gradient $\widetilde{g}$ and the Hessian $\widetilde{H}$ of $\widetilde{f}$ at a point $(x, y)$ satisfy

$$
\tilde{g}=\left(\begin{array}{c}
-A^{T} S^{-1} \mathbf{1} \\
\frac{1}{1-y}+b^{T} S^{-1} \mathbf{1}
\end{array}\right), \quad \tilde{H}=\left(\begin{array}{cc}
A^{T} S^{-2} A & -A^{T} S^{-2} b \\
\left(-A^{T} S^{-2} b\right)^{T} & \frac{1}{(1-y)^{2}}+b^{T} S^{-2} b
\end{array}\right),
$$

where the Hessian inverse can be computed using Schur complement and is equal to

$$
\begin{aligned}
\tilde{H}^{-1}= & \left(\begin{array}{cc}
\left(A^{T} S^{-2} A\right)^{-1} & 0 \\
0 & 0
\end{array}\right) \\
& +\frac{1}{\frac{1}{(1-y)^{2}}-\gamma}\left(\begin{array}{c}
\left(A^{T} S^{-2} A\right)^{-1} A^{T} S^{-2} b \\
1
\end{array}\right)\left(\begin{array}{c}
\left(A^{T} S^{-2} A\right)^{-1} A^{T} S^{-2} b \\
1
\end{array}\right),
\end{aligned}
$$

where $\gamma=b^{T} S^{-2} b-\left(A^{T} S^{-2} b\right)^{T}\left(A^{T} S^{-2} A\right)^{-1} A^{T} S^{-2} b$.

In what follows, we adapt the notation of [10]: we write $\|u\|_{x}$ for the local norm of $v$ at $x$, where the norm is induced by the intrinsic inner product at $x \in D_{f}$ arising from the strongly non-degenerate self-concordant function $f: D_{f} \rightarrow \mathbb{R}$, namely $\|u\|=$ $u^{T} \nabla^{2} f(x) u$. First we show that, for $\theta$ large enough, any segment of $\mathcal{P}$ corresponding to $v \in[\underline{v}, \bar{v}]$ can be well approximated by a suitably chosen segment of $\widetilde{\mathcal{P}}$; in doing so we will manufacture a good surrogate for a point $(\tilde{x}, \tilde{y})(v) \in \widetilde{\mathcal{P}}$ from $x\left(\frac{1}{2}(v+\right.$ $\left.\left.\sqrt{v^{2}-v / \theta}\right)\right) \in \mathcal{P}$.

Proposition 2.1 Let $v \in[\underline{v}, \bar{v}]$ and $M$ be such that $\left|b^{T} S^{-1} \mathbf{1}\right|, \sqrt{|\gamma|} \leq M$ for all $(y x(v), y), \frac{1}{2} \leq y \leq 1, v \in[\underline{v}, \bar{v}]$. If $\theta$ is chosen large enough to satisfy $\theta \geq$ $\frac{1}{v} \max \{1, \sqrt{82} M-1\}$, then, for $y=1-\frac{1}{1+\theta v}$, the point $(y x(v), y)$ approximates $(\widetilde{x}, \tilde{y})(v / y)$, namely

$$
\left\|\left(\begin{array}{c}
\tilde{x} \\
\tilde{y}
\end{array}\right)(v / y)-\left(\begin{array}{c}
y x(v) \\
y
\end{array}\right)\right\|_{(y x(v), y)} \leq \frac{\sqrt{82}}{3} \frac{M}{1+\theta v} .
$$

Remark 2.2 $M<\infty$ since the portion of the central path $\mathcal{P}$ corresponding to any finite interval $[\underline{v}, \bar{v}]$ lies in the interior of $P$. 
Remark 2.3 With $y=1-\frac{1}{1+\theta v}$, the function $v \mapsto \frac{v}{y}$ is increasing for $v \geq 0$ and hence is invertible with its inverse being $v \mapsto \frac{1}{2}\left(v+\sqrt{v^{2}-v / \theta}\right)$.

Remark 2.4 Recalling that $\tilde{f}$ is strongly non-degenerate self-concordant, and, therefore, an open unit ball in the local norm at $(x, y) \in \operatorname{int} \widetilde{P}$ satisfies $B_{(x, y)}((x, y), 1) \subset$ int $\widetilde{P}$, where $\widetilde{P}$ is bounded by assumption, the proposition implies that $(\widetilde{x}, \widetilde{y})(v) \rightarrow$ $(x(v), 1)$ as $\theta \rightarrow \infty$. Moreover, the convergence is uniform over any finite segment $[\underline{v}, \bar{v}]$ of $\mathcal{P}$.

Proof We rely on the fact that the Newton's method iterates exhibit local quadratic convergence to the central path for linear optimization [11], which may be rephrased in a more general setting of strongly non-degenerate self-concordant functions [10]. For concreteness, we use an intermediate statement in the proof of Proposition 2.2.8 of [10]: let $n(x)=-\nabla^{2} f(x)^{-1} \nabla f(x)$ be the Newton step for strongly nondegenerate self-concordant function $f: D_{f} \rightarrow \mathbb{R}$; if $\|n(x)\|_{x} \leq \frac{1}{9}$, then $f$ has a minimizer $z$ and $\|x-z\|_{x} \leq 3\|n(x)\|_{x}$.

Consider the Newton step for minimizing $v^{\prime}(c,-\theta)^{T}(x, y)+\widetilde{f}(x, y)$ and evaluate (the square of) its local norm at $(y x(v), y)$, where $y=1-\frac{1}{1+\theta v}$ and $v^{\prime}=\frac{v}{y}$ :

$$
\begin{aligned}
& \left.\left(v^{\prime}\left(\begin{array}{c}
c \\
-\theta
\end{array}\right)+\widetilde{g}\right)^{T} \tilde{H}^{-1}\left(v^{\prime}\left(\begin{array}{c}
c \\
-\theta
\end{array}\right)+\widetilde{g}\right)\right|_{(y x(v), y)} \\
& =\left.\left(\begin{array}{c}
\frac{v}{y} c+\frac{1}{y} \nabla f \\
\frac{-v}{y} \theta+\frac{1}{1-y}+b^{T} S^{-1} \mathbf{1}
\end{array}\right)^{T} \tilde{H}^{-1}\left(\begin{array}{c}
\frac{v}{y} c+\frac{1}{y} \nabla f \\
\frac{-v}{y} \theta+\frac{1}{1-y}+b^{T} S^{-1} \mathbf{1}
\end{array}\right)\right|_{(y x(v), y)}
\end{aligned}
$$

and since $x(v) \in \mathcal{P}$

$$
\begin{aligned}
& =\left.\left(\frac{-v}{y} \theta+\frac{1}{1-y}+b^{T} S^{-1} \mathbf{1}\right)^{T} \tilde{H}^{-1}\left(\frac{-v}{y} \theta+\frac{1}{1-y}+b^{T} S^{-1} \mathbf{1}\right)\right|_{(y x(v), y)} \\
& =\left.\left(\frac{-v}{y} \theta+\frac{1}{1-y}+b^{T} S^{-1} \mathbf{1}\right)^{2} \frac{1}{\frac{1}{(1-y)^{2}}+\gamma}\right|_{(y x(v), y)}
\end{aligned}
$$

and by the choice of $y$ and $\theta$

$$
=\left.\frac{\left(b^{T} S^{-1} \mathbf{1}\right)^{2}}{\frac{1}{(1-y)^{2}+\gamma}}\right|_{(y x(v), y)} \leq \frac{M^{2}}{(1+\theta v)^{2}-M^{2}} \leq \frac{82}{81}\left(\frac{M}{1+\theta v}\right)^{2} \leq \frac{1}{81} .
$$

Now, since the size of the Newton step for minimizing $v^{\prime}(c,-\theta)^{T}(x, y)+\widetilde{f}(x, y)$ measured with respect to the local norm at $(y x(v), y)$ is indeed $\leq \frac{1}{9}$, the statement of the proposition immediately follows.

We present one useful proposition. Roughly speaking, it states that two similar curves may not differ greatly in their total curvatures either. 
Proposition 2.2 Let $\psi:[\alpha, \beta] \rightarrow \mathbb{R}^{d}$ be a $C^{2}((\alpha-\varepsilon, \beta+\varepsilon))$ map for some $\varepsilon>$ 0 with a nonzero derivative in $[\alpha, \beta]$, and $\left\{\phi^{j}\right\}_{j=0,1, \ldots}$ be a sequence of $C^{2}((\alpha-$ $\varepsilon, \beta+\varepsilon)$ ) functions with nonzero derivatives in $[\alpha, \beta]$ that converge to $\psi$ pointwise as $j \rightarrow \infty$, i.e., $\phi^{j}(t) \rightarrow \psi(t)$ for all $t \in[\alpha, \beta]$. Then the total curvature of $\psi$ is bounded from above by the infimum limit of the total curvature of $\phi^{j}$ over all $j$.

For compactness, we sketch an elementary proof of this proposition in Sect. 3. For a detailed exposition of a very similar argument, see [3].

Next we argue that the total curvature of any finite segment $[\underline{v}, \bar{v}]$ of the central path $\mathcal{P}$ may not be much less than the total curvature of $[\underline{v}, \bar{v}]$-segment of $\widetilde{\mathcal{P}}$. Indeed, this follows from Proposition 2.2, where the pointwise convergence of two paths follows from Remark 2.4. Note that the gradient to the central path does not vanish, since a point $x(v) \in \mathcal{P}$ is the minimizer for $v c^{T} x+f(x)$ and hence must satisfy $v c+\nabla f(x)=0$, and the derivative with respect to $v$ satisfies $\dot{x}(v)=-\nabla^{2} f(x)^{-1} c \neq 0$, recalling that, under our assumptions, $\nabla^{2} f(x)$ is nonsingular. Using a similar argument, one can show $\mathcal{P}$ is $C^{2}$ with respect to $v$, although a particular parametrization of $\mathcal{P}$ is not important, since it is already well known that $\mathcal{P}$ is real-analytic [12]. Same considerations apply to $\widetilde{\mathcal{P}}$.

In turn, the total curvature of $\mathcal{P}$ may be arbitrarily well approximated by the total curvature of a suitably chosen finite segment of the path by letting $\underline{v} \rightarrow 0$ and $\bar{v} \rightarrow \infty$. The later follows from the finiteness of the total curvature of $\mathcal{P}$ established in [2] or intuitively from the fact that $\mathcal{P}$ is asymptotically straight as $v \rightarrow 0$ and $v \rightarrow \infty$.

Finally, we can summarize our findings in the following lemma.

Lemma 2.1 With the construction above, as $\theta \rightarrow \infty$, we have $\liminf \lambda^{(c,-\theta)}(\widetilde{P})>$ $\lambda^{c}(P)$.

The proof that $\liminf \lambda^{(c,-\theta)}(\widetilde{P}) \geq \lambda^{c}(P)$ easily follows from the Remark 2.4 and Proposition 2.2. For strict inequality, using the techniques in $[3,4]$, one can show that just before $\widetilde{\mathcal{P}}$ "starts to converge" to $\mathcal{P}, \widetilde{\mathcal{P}}$ is bound to make a first sharp turn which in the limit will contribute to $\pi / 2$ additional total curvature for this path.

Now, inductively increasing the dimension and the number of inequalities by 1 and carefully using the limit argument in the above, the same construction gives a sequence of polytopes $\widetilde{P}=\widetilde{P}_{1}, \widetilde{P}_{2}, \ldots, \widetilde{P}_{n-2 d}$ satisfying $\lambda^{c}(P) \leq \lambda^{(c,-\theta)}\left(\widetilde{P}_{1}\right) \leq$ $\cdots \leq \lambda^{(c,-\theta, \ldots)}\left(\widetilde{P}_{n-2 d}\right)$. Since $\widetilde{P}_{n-2 d}$ is defined by $2 n-2 d$ inequalities in dimension $n-d$, we have $\lambda^{(c,-\theta, \ldots)}\left(\widetilde{P}_{n-2 d}\right) \leq 2 K(n-d)$. This implies that, for $n>2 d$, $\lambda^{c}(P) \leq 2 K(n-d)$, that is, $\Lambda(d, n)=\mathcal{O}(n)$.

\section{Sketch of the Proof of Proposition 2.2}

Observe that without loss of generality we may assume that $\psi$ is parameterized by the arc length: a short computation shows that if $\dot{\psi} \neq 0$, then the second derivative of the arc length parametrization of the curve is continuous, since $\dot{\psi}_{\text {arc }}(t)=\psi\left(l^{-1}(t)\right)_{t}^{\prime}=$ $\dot{\psi}\left(l^{-1}(t)\right) \cdot\left(l^{-1}(t)\right)_{t}^{\prime}=\frac{\dot{\psi}\left(l^{-1}(t)\right)}{\left\|\dot{\psi}\left(l^{-1}(t)\right)\right\|}$, and consequently $\ddot{\psi}_{\text {arc }} \circ l=\frac{\ddot{\psi}\|\dot{\psi}\|^{2}-\dot{\psi}\left(\ddot{\psi}^{T} \dot{\psi}\right)}{\|\dot{\psi}\|^{3}}$. In 
Fig. 2 Illustration of the proof of Proposition 2.2

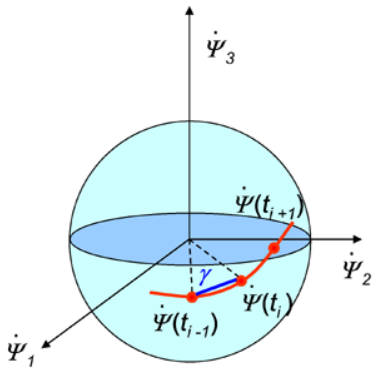

(a)

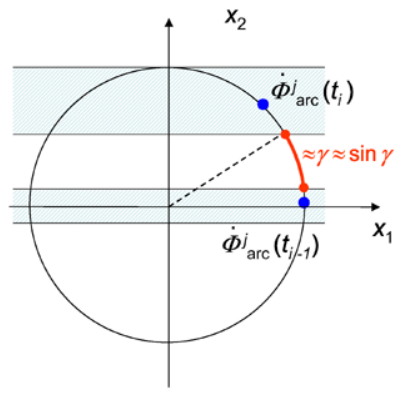

(c)

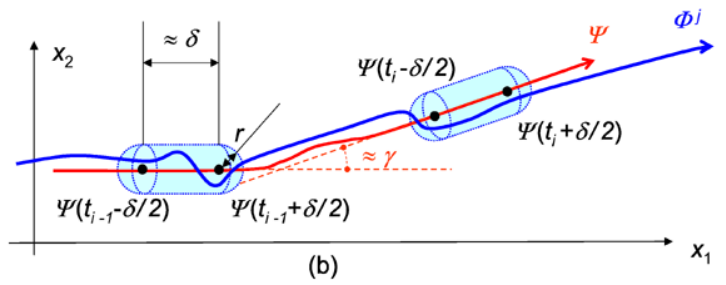

(b)

what follows, we remove the arc subscript from $\psi$ to shorten the notation and write $\alpha$ for $l(\alpha)$ and $\beta$ for $l(\beta)$. Note that the total curvature of $\psi$ corresponds to the length of the gradient curve $\dot{\psi}$ between the points $\dot{\psi}(\alpha)$ and $\dot{\psi}(\beta)$, which in turn belong to the unit $n$-sphere.

First, we argue that the total curvature of $\psi$ can be arbitrarily well approximated by the sum of chordal distances between pairs $\left(\dot{\psi}\left(t_{i-1}\right), \dot{\psi}\left(t_{i}\right)\right), \sum_{i=1}^{N} \| \dot{\psi}\left(t_{i}\right)-$ $\dot{\psi}\left(t_{i-1}\right) \|$, where $t_{0}=\alpha<t_{1}<t_{2}<\cdots<t_{N}=\beta$ as long as $\max _{i=1, \ldots, N}\left(t_{i}-t_{i-1}\right) \rightarrow$ 0 , see Fig. 2(a). Trivially $\int_{\alpha}^{\beta}\|\ddot{\psi}(t)\| d t=\sum_{i=1}^{N} \int_{t_{i-1}}^{t_{i}}\|\ddot{\psi}(t)\| d t$, and observe that

$$
\begin{aligned}
& \left|\int_{t_{i-1}}^{t_{i}}\|\ddot{\psi}(t)\| d t-\int_{t_{i-1}}^{t_{i}} \frac{\left\|\dot{\psi}\left(t_{i}\right)-\dot{\psi}\left(t_{i-1}\right)\right\|}{t_{i}-t_{i-1}} d t\right| \\
& \quad \leq \int_{t_{i-1}}^{t_{i}}\left|\|\ddot{\psi}(t)\|-\frac{\left\|\dot{\psi}\left(t_{i}\right)-\dot{\psi}\left(t_{i-1}\right)\right\|}{t_{i}-t_{i-1}}\right| d t \\
& \quad \leq \int_{t_{i-1}}^{t_{i}}\left\|\ddot{\psi}(t)-\frac{\dot{\psi}\left(t_{i}\right)-\dot{\psi}\left(t_{i-1}\right)}{t_{i}-t_{i-1}}\right\| d t \\
& \quad=\int_{t_{i-1}}^{t_{i}}\left\|\ddot{\psi}(t)-\left(\begin{array}{c}
\ddot{\psi}_{2}\left(\bar{t}_{2}\right) \\
\vdots \\
\ddot{\psi}_{n}\left(\bar{t}_{n}\right)
\end{array}\right)\right\| d t \leq \int_{t_{i-1}}^{t_{i}} M t d t=\frac{M\left(t_{i}-t_{i-1}\right)^{2}}{2}
\end{aligned}
$$

with $t_{i-1} \leq \bar{t}_{i} \leq t_{i}$ for all $i$, where the second inequality follows from the triangle inequality, the first equality follows from the Intermediate-Value Theorem, and the last inequality is implied by the Lipschitz continuity of the second derivative of $\psi$ on the compact set, where the Lipschitz constant is denoted by $M$. So, up to a quadratic error term the curvature contribution over the segment $\left[t_{i-1}, t_{i}\right]$ can be approximated 
by the length of the linear segment connecting the starting point $\dot{\psi}\left(t_{i-1}\right)$ and ending point $\dot{\psi}\left(t_{i}\right)$ with both points on the unit sphere, implying a linear error term for the total curvature approximation over $[\alpha, \beta]$. In particular, we may consider the partitioning $t_{0}=\alpha<t_{1}<t_{2}<\cdots<t_{N}=\beta$, where each $\left\|\dot{\psi}\left(t_{i}\right)-\dot{\psi}\left(t_{i+1}\right)\right\|=\gamma$ for some small fixed $\gamma$.

Note that any partitioning of $[\alpha, \beta]$ trivially gives a lower bound of $\sum_{i=1}^{N} \| \dot{\phi}^{j}\left(t_{i}\right)-$ $\dot{\phi}^{j}\left(t_{i-1}\right) \|$ on the total curvature of $\phi^{j}$ for all $j$. Also, note that since $\phi^{j}(t) \rightarrow \psi(t)$ as $j \rightarrow \infty$ on a compact set $[\alpha, \beta]$, the convergence is uniform.

Next we claim that, for $j$ large enough, the changes in the first derivative of $\phi^{j}$ in the neighboring segment to $\psi\left(\left[t_{i-1}, t_{i}\right]\right)$ is at least almost as large as the change in the derivative of $\psi$ itself over $\left[t_{i-1}, t_{i}\right]$, namely is bounded below by $\approx\left\|\dot{\psi}\left(t_{i}\right)-\dot{\psi}\left(t_{i+1}\right)\right\|=\gamma$. If this is true, then since $\sum_{i=1}^{N}\left\|\dot{\phi}^{j}\left(t_{i}\right)-\dot{\phi}^{j}\left(t_{i-1}\right)\right\|$ gives a lower bound on the total curvature of $\phi^{j}$, the argument would be complete. To show that the former is indeed the case, consider $\delta<1 / 2 \max _{i=1, \ldots, N}\left(t_{i}-t_{i-1}\right)$ and small enough so that $\dot{\psi}\left(t_{i}\right) \approx \frac{\left.\psi\left(t_{i}+\delta / 2\right)-\psi\left(t_{i}-\delta / 2\right)\right)}{\delta}$, where the approximation error is quadratic in $\delta$ and uniform for all $i$ (again, this can be achieved since $\psi$ is $C^{2}$ over a compact). Since the convergence $\phi^{j}(t) \rightarrow \psi(t)$ is uniform, for any $r>0$, there exists $J$ such that, for all $j \geq J$, we have $\left|\phi^{j}(t)-\psi(t)\right|<r$ for all $t \in[\alpha, \beta]$. Pick $J$ such that $r \ll \delta \sin \gamma$. Consider two cylindrical tubes with rounded bases around the segments $\left[\psi\left(t_{i-1}-\delta / 2\right), \psi\left(t_{i-1}+\delta / 2\right)\right]$ and $\left[\psi\left(t_{i}-\delta / 2\right), \psi\left(t_{i}+\delta / 2\right)\right]$; each tube is a union of a cylinder of height $\approx \delta$ and radius $r$ with base centers at $\psi\left(t_{k}-\delta / 2\right)$ and $\psi\left(t_{k}+\delta / 2\right), k=i-1$ and $i$, respectively, and two $r$-balls with the same centers. Align the coordinate system so that the first coordinate is collinear with the vector $\left(\psi\left(t_{i-1}-\delta / 2\right), \psi\left(t_{i-1}+\delta / 2\right)\right)$, and the second coordinate is chosen so that, together with the first coordinate axis, it spans a hyperplane parallel to $\left[\psi\left(t_{i}-\delta / 2\right), \psi\left(t_{i}+\delta / 2\right)\right]$, with the first two coordinates of $\psi\left(t_{i}\right)$ positive, see Fig. 2(b). The following two inequalities should be interpreted as true up to higherorder error terms. Note that by the Intermediate-Value Theorem there exists $\underline{\tau}$ such that $\left|\left(\dot{\phi}_{\operatorname{arc}}^{j}(\underline{\tau})\right)_{2}\right| \leq \frac{2 r}{\delta-2 r}$ : considering the tube around $\left[\psi\left(t_{i-1}-\delta / 2\right), \psi\left(t_{i-1}+\delta / 2\right)\right]$, note that for $\phi_{\text {arc }}^{j}$ to traverse the tube will take at least $\delta-2 r$ change of the arc length parameter, while, at the same time, its second coordinate will change by at most $2 r$. Similarly, considering the second tube, we conclude that there exists $\bar{\tau}$ such that $\left|\left(\dot{\phi}_{\operatorname{arc}}^{j}(\bar{\tau})\right)_{2}\right| \geq \frac{\delta \sin \gamma-2 r}{\delta+2 r}$. Clearly, with $r$ small enough, or equivalently, $j$ large enough, $\left\|\dot{\phi}_{\text {arc }}^{j}\left(t_{i}\right)-\dot{\phi}_{\text {arc }}^{j}\left(t_{i-1}\right)\right\|$ is at least $\gamma$ up to the higher-order terms, since we consider the shortest distance between two points $\dot{\phi}_{\operatorname{arc}}^{j}(\underline{\tau})$ and $\dot{\phi}_{\operatorname{arc}}^{j}(\bar{\tau})$ on the $n$-unit sphere, see Fig. 2(c).

Acknowledgements Research supported by an NSERC Discovery grant, by a MITACS grant, and by the Canada Research Chair program.

\section{References}

1. Dantzig, G.: Linear Programming and Extensions. Princeton University Press, Princeton (1963)

2. Dedieu, J.-P., Malajovich, G., Shub, M.: On the curvature of the central path of linear programming theory. Found. Comput. Math. 5, 145-171 (2005) 
3. Deza, A., Terlaky, T., Zinchenko, Y.: Central path curvature and iteration-complexity for redundant Klee-Minty cubes. In: Gao D. and Sherali H. (eds.) Advances in Mechanics and Mathematics, vol. III, pp. 215-248 (2007)

4. Deza, A., Terlaky, T., Zinchenko, Y.: Polytopes and arrangements: diameter and curvature. Oper. Res. Lett. 36-2, 215-222 (2008)

5. Fritzsche, K., Holt, F.: More polytopes meeting the conjectured Hirsch bound. Discrete Math. 205, 77-84 (1999)

6. Holt, F., Klee, V.: Many polytopes meeting the conjectured Hirsch bound. Discrete Comput. Geom. 20, 1-17 (1998)

7. Grünbaum, B.: Convex Polytopes. In: Kaibel, V., Klee, V., Ziegler, G. (eds.) Graduate Texts in Mathematics. vol. 221. Springer, Berlin (2003)

8. Kalai, G., Kleitman, D.: A quasi-polynomial bound for the diameter of graphs of polyhedra. Bull. Am. Math. Soc. 26, 315-316 (1992)

9. Klee, V., Walkup, D.: The $d$-step conjecture for polyhedra of dimension $d<6$. Acta Math. 133, 53-78 (1967)

10. Renegar, J.: A Mathematical View of Interior-Point Methods in Convex Optimization. SIAM, Philadelphia (2001)

11. Roos, C., Terlaky, T., Vial, J.-Ph.: Interior Point Methods for Linear Optimization. Springer, Berlin (2006)

12. Sonnevend, G., Stoer, J., Zhao, G.: On the complexity of following the central path of linear programs by linear extrapolation II. Math. Program. 52(1-3), 527-553 (1991)

13. Spivak, M.: Comprehensive Introduction to Differential Geometry. Publish or Perish, Houston (1990)

14. Ziegler, G.: Lectures on Polytopes, Graduate Texts in Mathematics, vol. 152. Springer, Berlin (1995) 